\title{
Eating Occasions, Obesity and Related Behaviors in Working Adults: Does it Matter When You Snack?
}

\author{
Wendy E. Barrington ${ }^{1,2,3, *}$ and Shirley A. A. Beresford ${ }^{2,3}$ \\ 1 Department of Child, Family, and Population Health Nursing, University of Washington, Seattle, \\ WA 98195, USA \\ 2 Department of Epidemiology, University of Washington, Seattle, WA 98195, USA; sberesfrd@uw.edu \\ 3 Cancer Prevention Program, Fred Hutchinson Cancer Research Center, Seattle, WA 98195, USA \\ * Correspondence: wendybar@uw.edu; Tel.: +1-206-616-6298
}

Received: 19 August 2019; Accepted: 27 September 2019; Published: 1 October 2019

check for updates

\begin{abstract}
Reported relationships between frequency, type, and timing of eating occasions and obesity-risk among adults are mixed while associations with obesogenic eating behaviors remain unexplored. The Physical Activity and Changes in Eating (PACE) study was a group-randomized controlled trial to prevent weight gain among 34 small worksites in Seattle from 2005-2009. Baseline surveys assessed body mass index (BMI), obesogenic eating behaviors (e.g., fast food and distracted-eating), and eating occasions (i.e., snacks and meals) among 2265 employees. BMI and waist circumference were measured on a subset $(n=567)$. Time-periods for analyses included: morning (12:00 a.m. to 10:59 a.m.), mid-day (11:00 a.m. to 4:29 p.m.), and evening (4:30 p.m. to 11:59 p.m.). Multilevel linear models estimated associations between snack timing, obesity, and related behaviors while adjusting for meal timing, gender, and worksite random effects. Greater morning snacking was associated with increased fruit and vegetable consumption, while greater evening snacking was associated with higher BMI, higher obesogenic dietary index (intake of fast food, French fries, and soft drinks), and higher percent time eating while distracted. Associations with mid-day snacking were mixed. Patterns of association were consistent across repeated and objective measures. Findings suggest that evening snacking is more detrimental to healthy weight compared to snacking at other times of day. Reducing evening snacks may be an important and simple message for population-level obesity prevention efforts.
\end{abstract}

Keywords: snacking; obesity; obesogenic; behaviors

\section{Introduction}

Obesity continues to threaten the health of U.S. adults-almost $40 \%$ are affected and obesity prevalence among women has continued to increase [1]. Alarmingly, obesity prevention efforts, including dietary modification, have largely been unsuccessful in maintaining long-term weight reduction [2]. Our understanding of what dietary interventions are most effective is limited not only by how diet has been measured, but also by what messages have been promoted for dietary change. Most studies have focused on communicating relationships between nutrient intake and obesity-risk. Focus has shifted recently towards understanding the composition of 'obesogenic' eating patterns and dietary behaviors. This approach is not only more easily communicated to the public in the form of dietary guidelines, but also accounts for how nutrients are consumed together as foods and meals [3].

Eating occasions is a term used to describe the ingestion of any type of meal or drink which can be further categorized by amount and type of food consumed as well as the time of day it was consumed [3]. Some [4,5], but not all, evidence supports associations between greater frequency of eating occasions and obesity [4,5]. However, our understanding of the relationships between 
type and timing (i.e., pattern) of eating occasions and obesity is less clear due to differences in how eating occasions are defined across studies [3,4]. Some studies pre-define the eating occasion (e.g., breakfast, morning snack, lunch, afternoon snack, dinner, evening snack, overnight) [3,4] for the respondent. Such definitions assume intake occurs at implied culturally-relevant times and usually provide examples of relevant foods for each type of eating occasion. Other studies may rely on other factors associated with the eating occasion (e.g., duration, timing, portion size, energy density, $\%$ contribution to daily energy intake) for the researcher to define the type of eating occasion $[3,4]$. For snacks in particular, some U.S. studies define these occasions by intake of specific foods that tend to be energy-dense [3,4]. Despite these methodological differences, several reviews consistently support associations between greater snacking frequency [4-8], as well as eating later in the day [6,9], with higher obesity risk. However, most studies have not evaluated the specific contribution of snacking while accounting for other eating occasions [3]. Such analyses could more closely model associations between what may be considered 'extra' eating occasions and obesity. Furthermore, understanding the relationship between snacking pattern and obesogenic behaviors is also warranted [4]. The effects of snacking may depend not just on the amount of food consumed, but behaviors that determine 'what' and 'how' food is consumed [10-12].

The Promoting Activity and Changes in Eating (PACE) study provides a unique opportunity to address these methodologic issues using a novel grid assessment method $[13,14]$ for measuring eating occasions and patterns. This method allows the respondent to categorize their own eating occasion as well as document when intakes occurred. The objective of this study, specifically, is to evaluate snacking patterns in relation to obesity (i.e., body mass index, waist circumference), and to obesity-related behaviors (i.e., fruit and vegetable consumption, obesogenic dietary index score, and distracted-eating). We hypothesize that that higher snacking, especially later in the day, will be associated with obesity among this sample of adults. Further, if their snacking patterns are associated with other obesogenic dietary behaviors like fast food or soda intake, this may support directing behavior change goals to reduce snacking, as a simple and more easily accepted strategy, in future obesity prevention interventions.

\section{Materials and Methods}

\subsection{Study Population}

The Promoting Activity and Changes in Eating (PACE) study was a large group-randomized weight-gain prevention trial among approximately 3000 individuals in 34 worksites in the Seattle Metropolitan area. Eligible worksites that employed between 40 and 350 workers were identified using U.S. Standard Identification Classification two-digit codes; worksite categories included: manufacturing, transportation or utilities, personal services, household and miscellaneous services, and non-classifiable establishments. Eligibility criteria included having: 1) a high proportion of sedentary employees; 2 ) a low turn-over rate during the previous two years; and 3) a low proportion of non-English speaking employees. Detailed description of the PACE study has been reported elsewhere [15].

All employees of participating worksites with fewer than 150 employees and a random subsample of 125 employees within worksites with greater than 150 employees were asked to complete a standard questionnaire assessing self-reported dietary and physical activity behaviors, height, weight, and demographic information at baseline (2005-2007). An independent sample of employees, derived in the same way, were invited to complete the follow-up questionnaire (2007-2009). At baseline, 3054 employees within 34 worksites provided survey data; a random 'intensive assessment' subsample of 622 employees was also invited to provide additional physical measures including measured height, weight, and waist circumference. The present analysis uses survey data from 2389 individuals without missing data from the eating occasion grid or gender at baseline. Of these individuals, 1151 provided survey data at 2-year follow-up as well as physical measurements as part of intensive assessment 
subsamples: 568 at baseline and 268 at follow-up. This study was approved by the Fred Hutchinson Cancer Research Center Institutional Review Board.

\subsection{Eating Occasions and Patterns}

The time and type of eating occasion were collected using a pattern matrix or grid adapted from the work of Berteus Forslund and colleagues [13,14]. In brief, respondents wrote in the time of every eating occasion and checked which type of meal that represented. The choices were: main meal (e.g., cooked dish, hearty soup with bread, Chef salad with bread, pizza); light meal/breakfast (e.g., cooked or cold cereals, simple soup, side salad); snack meal (e.g., cookie, slice of cake/pie, energy bar, fruit, candy, ice cream); or drink only (e.g., coffee, tea, soft drink, juice, milk, beer, wine). Respondents were not limited in the number of entries they could submit on the grid. Our adaptation of the grid is included as Supplementary Material.

\subsubsection{Snack Frequency}

The distribution of snack times over the day was visually examined to identify natural cut-points. Based on this approach, three major snack time periods were identified including: morning (12:00 a.m. to 10:59 a.m.), mid-day (11:00 a.m. to 4:29 p.m.), and evening (4:30 p.m. to 11:59 p.m.). The number of snack occasions was summed within each snack period to obtain the frequency and grouped into frequency categories (i.e., ' 0 ', ' 1 ', ' $2+$ ').

\subsubsection{Intake of Main Meal}

Presence or absence of a main or light meal within each of the distinct snack periods referenced above resulted in three binary variables: morning meal, mid-day meal, and evening meal.

\subsection{Outcome Variables}

\subsubsection{Body Mass Index (BMI)}

At both baseline and follow-up, height, and weight were assessed via self-report on the survey, and were measured in the intensive assessment subsample by trained study personnel using a stadiometer and scale, respectively. BMI was calculated as weight $(\mathrm{kg})$ divided by height $\left(\mathrm{m}^{2}\right)$ using both self-reported and physically measured data in two separate variables for these analyses.

\subsubsection{Waist Circumference}

Waist circumference was assessed via physical measurement during the same intensive assessment within worksites by study personnel at baseline and follow-up. Values are reported in $\mathrm{cm}$.

\subsubsection{Fruit and Vegetable Servings Per Day}

Increased daily servings of fruits and vegetables is a behavior promoted heavily by public health professionals as it has been found to be inversely associated with obesity [16]. Components of fruit and vegetable consumption were assessed using the National Cancer Institute's seven-item 5-a-Day fruit and vegetable assessment tool [17]. Total servings of fruit and vegetables was calculated by summing all components except frequency of eating fried potatoes or French fries.

\subsubsection{Index of Obesogenic Dietary Behaviors Per Week}

Using reduced rank regression, several dietary behaviors were identified that best predicted obesity among PACE participants [18]. The index is a simple average of weekly frequency of three intakes: french fries, soft drinks, and fast food meals. Frequency of eating fried potatoes or french fries was assessed by one component of the NCI 5-a-Day fruit and vegetable assessment tool [17]. Frequency of fast-food meals was assessed by the item, "Thinking about how often you eat out, how 
many times in a week or month do you eat breakfast, lunch, or dinner in a place such as McDonald's $\mathrm{s}^{\circledR}$, Burger King ${ }^{\circledR}$, Wendy's ${ }^{\circledR}$, Arby's $^{\circledR}$, Pizza Hut ${ }^{\circledR}$, or Kentucky Fried Chicken ${ }^{\circledR}$ ?") $[19,20]$. Responses were given as times per week or times per month. All responses were converted to times per week. Average weekly soft-drink intake was assessed via the item, "How often do you drink soft drinks or soda pop (regular or diet)?" [21]. Response options were: "Never", "Less than once a week", "About once a week", "2-5 times per week", "About once a day", and "2 or more times per day."

\subsubsection{Task-Eating}

This construct connotes a level of distraction, or lack of eating awareness or mindfulness, which has also been linked to obesity [22,23]. Task-eating was assessed via single-item, "How often do you eat food (meals or snacks) while doing another activity, for example, watching TV, working at a computer, reading, driving, or playing video games?" [10]. Response options were on a five-point Likert scale ranging from $1=$ 'Never' to $5=$ 'Always'.

\subsection{Covariates}

Individual-level factors in models were: age, gender, race/ethnicity (where 'Other' consolidates smaller racial/ethnic subgroups including: Native Alaskan/American Indian and Pacific Islander/Native Hawaiian groups), and education. To further adjust for lifestyle differences, models also included adjustment for manual (i.e., machine operators, mechanics/technicians, service workers, tradesmen, or laborer) or non-manual occupation and for leisure-time physical activity of at least 10 minutes in duration via the Godin-Shephard Leisure-Time Physical Activity Questionnaire [24]. The questionnaire estimates the frequency of exercise bouts (i.e., vigorous, moderate, and light), multiplies each bout by the corresponding metabolic equivalents (i.e., 9, 5, and 3), and then sums these components to create an intensity-weighted score (i.e., leisure score index) that corresponds to a metabolic equivalent of task frequency per week [24].

\subsection{Statistical Analysis}

To examine associations between snacking and obesity-related outcomes, frequency categories of snack occasions was regressed against outcome variables adjusted for covariates and presence/absence of a main or light meal for the corresponding snack period. Continuous outcome variables were log-transformed to account for skewness during analyses and marginal means were calculated for frequency categories within snack periods. All analyses were conducted using Stata SE version 13.0 (StataCorp, College Station, TX, USA).

\section{Results}

Baseline demographic and eating occasion data among PACE participants are summarized in Table 1 . The mean age of participants was 43 years and participants were primarily white, had greater than a high school education, worked in white- or pink-collar jobs (i.e., non-manual occupation), and were sufficiently active according to an established cut-point ( $\geq 24$ leisure score index) for the Godin Shepard Leisure-Time Physical Activity Questionnaire [25]. Participants reported eating about 2 main meals, 1 light meal, 1.5 snacks, and 1 solo drink per day. About 80 percent of respondents reported snacking at least once. In the morning, $66.6 \%$ reported eating a meal whereas meal intake was higher during later time-periods ( $90.5 \%$ and $93.9 \%$, respectively).

Correlations between eating occasions, dietary behaviors, and obesity outcomes at baseline are presented in Table 2 . There was virtually no correlation between snacking variables nor meal variables. However, there was a modest negative correlation between morning snacking and morning meals and between mid-day snacking and mid-day meals while a modest correlation between morning meals and mid-day snacking was evident. Among dietary behaviors, there was modest negative correlation of fruit and vegetable intake with the obesogenic dietary index as well as with distracted eating. Conversely, both self-reported and measured obesity variables were highly correlated. 
Table 1. Summary of Baseline Demographic and Eating Occasion Variables Over Time Among Promoting Activity and Changes in Eating (PACE) Participants, 2005-2009.

\begin{tabular}{|c|c|c|c|c|c|c|c|c|}
\hline & \multicolumn{4}{|c|}{ Baseline } & \multicolumn{4}{|c|}{ Follow-Up } \\
\hline & \multicolumn{2}{|c|}{ Total } & $\begin{array}{l}\text { In } \\
\text { Ass } \\
\text { Sul }\end{array}$ & $\begin{array}{l}\text { ive } \\
\text { nent } \\
\text { iple }\end{array}$ & \multicolumn{2}{|c|}{ Total } & \multicolumn{2}{|c|}{$\begin{array}{c}\text { Intensive } \\
\text { Assessment } \\
\text { Subsample }\end{array}$} \\
\hline & \multicolumn{2}{|c|}{$(\mathrm{N}=34 ; n=2389)$} & \multicolumn{2}{|c|}{$(\mathrm{N}=34 ; n=568)$} & \multicolumn{2}{|c|}{$(\mathrm{N}=33 ; n=1151)$} & \multicolumn{2}{|c|}{$(\mathrm{N}=28 ; n=268)$} \\
\hline & $n$ & $\%^{a}$ & $n$ & $\%^{a}$ & $n$ & $\%^{a}$ & $n$ & $\%^{a}$ \\
\hline $\begin{array}{l}\text { Age (years) } \\
\text { Gender }\end{array}$ & 43.0 & 0.7 & 44.3 & 0.7 & 44.3 & 0.6 & 45.7 & 0.9 \\
\hline Men & 1141 & 47.4 & 230 & 40.4 & 529 & 46.0 & 97 & 36.2 \\
\hline $\begin{array}{l}\text { Women } \\
\text { Race }\end{array}$ & 1248 & 51.9 & 338 & 59.6 & 622 & 54.0 & 171 & 62.8 \\
\hline White & 1845 & 77.2 & 466 & 82.0 & 886 & 77.0 & 219 & 81.7 \\
\hline Hispanic/Latino & 124 & 5.2 & 33 & 5.8 & 59 & 5.1 & 18 & 6.7 \\
\hline African American & 70 & 2.9 & 25 & 4.4 & 34 & 3.0 & 8 & 3.0 \\
\hline Asian & 236 & 9.9 & 26 & 4.6 & 122 & 10.6 & 16 & 6.0 \\
\hline Other & 80 & 3.4 & 15 & 2.6 & 36 & 3.1 & 6 & 2.1 \\
\hline Missing & 34 & 1.4 & 3 & 0.5 & 14 & 1.2 & 1 & 0.4 \\
\hline \multicolumn{9}{|l|}{ Education } \\
\hline$<$ HS, HS diploma or GED certificate & 368 & 15.4 & 58 & 10.2 & 192 & 16.7 & 31 & 11.6 \\
\hline Some college or technical college & 827 & 34.6 & 237 & 41.7 & 412 & 35.8 & 103 & 38.4 \\
\hline College graduate & 820 & 34.3 & 190 & 33.5 & 379 & 32.9 & 96 & 35.8 \\
\hline Post-graduate & 364 & 15.2 & 83 & 14.6 & 162 & 14.1 & 37 & 13.8 \\
\hline Missing & 10 & 0.4 & 0 & 0.0 & 6 & 0.5 & 1 & 0.4 \\
\hline \multicolumn{9}{|l|}{ Manual occupation } \\
\hline No & 1874 & 77.9 & 493 & 86.8 & 889 & 77.2 & 229 & 85.5 \\
\hline Yes & 508 & 21.1 & 74 & 13.0 & 251 & 21.8 & 37 & 13.8 \\
\hline Missing & 24 & 1.0 & 1 & 0.2 & 11 & 1.0 & 2 & 0.8 \\
\hline $\begin{array}{l}\text { Leisure-time PA (METs per week) } \\
\text { Eating Occasions }\end{array}$ & 31.1 & 29.2 & 28.7 & 22.8 & 29.8 & 25.5 & 28.5 & 22.0 \\
\hline Main meals & 1.7 & 0.6 & 1.7 & 0.6 & 1.7 & 0.6 & 1.7 & 0.5 \\
\hline Light meals & 1.0 & 0.7 & 1.0 & 0.7 & 1.0 & 0.7 & 1.0 & 0.8 \\
\hline Snacks & 1.4 & 1.1 & 1.6 & 1.1 & 1.5 & 1.1 & 1.6 & 1.1 \\
\hline Drink only & 0.8 & 1.0 & 1.0 & 1.1 & 0.8 & 1.0 & 1.0 & 1.1 \\
\hline
\end{tabular}

N, number of worksites; n, number of participants; HS, high school; GED, general education development; PA, physical activity; MET, metabolic equivalent of task. a Percentages may not sum to 100 due to rounding error. ${ }^{b}$ Values are expressed as mean (standard error).

Table 2. Spearman's Correlations Between Eating Occasions, Dietary Behaviors, and Obesity Outcomes Among Promoting Activity and Changes in Eating (PACE) Intensive Assessment Participants at Baseline, 2005-2007.

\begin{tabular}{lllllllllllll}
\hline & $\mathbf{1}$ & $\mathbf{2}$ & $\mathbf{3}$ & $\mathbf{4}$ & $\mathbf{5}$ & $\mathbf{6}$ & $\mathbf{7}$ & $\mathbf{8}$ & $\mathbf{9}$ & $\mathbf{1 0}$ & $\mathbf{1 1}$ & $\mathbf{1 2}$ \\
\hline 1. Morning snack & 1.00 & & & & & & & & & & & \\
2. Mid-day snack & 0.10 & 1.00 & & & & & & & & & \\
3. Evening snack & 0.02 & -0.02 & 1.00 & & & & & & & & \\
4. Morning meal & -0.18 & 0.19 & 0.01 & 1.00 & & & & & & & \\
5. Mid-day meal & 0.07 & -0.12 & 0.07 & 0.09 & 1.00 & & & & & & \\
6. Evening meal & -0.05 & 0.06 & -0.13 & -0.03 & -0.04 & 1.00 & & & & & \\
7. BMI (self-report) & -0.06 & -0.07 & 0.13 & -0.04 & -0.03 & -0.05 & 1.00 & & & & \\
8. Fruits and vegetables & 0.15 & 0.19 & -0.02 & 0.24 & 0.13 & 0.07 & -0.20 & 1.00 & & & \\
9. Dietary index & 0.04 & -0.12 & 0.15 & -0.19 & 0.04 & 0.01 & 0.34 & -0.26 & 1.00 & & \\
10. Distracted eating & 0.07 & 0.06 & 0.16 & -0.09 & -0.03 & -0.10 & 0.08 & -0.11 & 0.11 & 1.00 & & \\
11. BMI (measured) & -0.04 & -0.06 & 0.13 & -0.07 & -0.02 & -0.05 & 0.96 & -0.19 & 0.35 & 0.06 & 1.00 & \\
12. Waist circumference & -0.06 & -0.14 & 0.10 & -0.10 & -0.06 & -0.01 & 0.84 & -0.20 & 0.32 & 0.01 & 0.86 & 1.00 \\
\hline \multicolumn{1}{c}{ a Among participants in intensive assessment subsample without missing data at baseline $(n=510)}$.
\end{tabular}


Outcome measures at baseline and follow-up are presented in Table 3. On average, participants were overweight/obese, did not meet the recommended number of five servings of fruit and vegetable per day, ate french fries, fast food, and soft-drinks about three times per week, and had high-levels of distracted eating (Table 3). Repeated measures at baseline and follow-up were highly correlated for all variables except distracted-eating.

Table 3. Summary of Outcomes at Baseline and 2-Year Follow-up Among Promoting Activity and Changes in Eating (PACE) Participants, 2005-2009.

\begin{tabular}{|c|c|c|c|c|c|}
\hline & \multicolumn{2}{|c|}{ Baseline } & \multicolumn{2}{|c|}{ Follow-Up } & \multirow[t]{2}{*}{ Spearman's Correlation Coefficient } \\
\hline & Mean & $S D$ & Mean & $S D$ & \\
\hline \multicolumn{6}{|l|}{ Self-Reported Measures ${ }^{a}$} \\
\hline $\operatorname{BMI}\left(\mathrm{kg} / \mathrm{m}^{2}\right)$ & 27.4 & 6.1 & 27.5 & 5.8 & 0.93 \\
\hline Fruit and vegetables (servings/day) & 3.2 & 2.2 & 3.5 & 2.2 & 0.63 \\
\hline Dietary index (times/week) & 2.9 & 3.0 & 2.5 & 2.8 & 0.76 \\
\hline High distracted-eating (\%) & 32.8 & 47.0 & 29.8 & 45.8 & 0.39 \\
\hline \multicolumn{6}{|l|}{ Objective Measures ${ }^{b}$} \\
\hline BMI $\left(\mathrm{kg} / \mathrm{m}^{2}\right)$ & 29.0 & 6.7 & 29.5 & 6.4 & 0.93 \\
\hline Waist circumference $(\mathrm{cm})$ & 90.3 & 15.9 & 87.7 & 15.1 & 0.90 \\
\hline
\end{tabular}

\footnotetext{
${ }^{\mathrm{a}}$ Among participants that provided survey data at baseline and follow-up $(n=1151) .{ }^{\mathrm{b}}$ Among participants in
} intensive assessment subsample at baseline and follow-up $(n=268)$.

Multilevel regression models were employed to examine associations between baseline frequency of snack occasions and outcome variables at baseline and follow-up. At baseline, greater morning snacking was associated with lower self-reported BMI, higher fruit and vegetable intake, and lower obesogenic dietary index score (Table 4). Greater mid-day snacking was associated with higher fruit and vegetable intake, lower obesogenic dietary index score, and higher levels of distracted-eating at baseline. Greater evening snacking was associated with higher self-reported BMI, higher obesogenic dietary index score, and higher levels of distracted-eating at baseline (Figure S1). At follow-up, higher baseline morning snacking was associated with higher fruit and vegetable intake (Table 5). Higher baseline mid-day snacking was associated with higher fruit and vegetable intake and lower obesogenic dietary index score at follow-up. Higher baseline evening snacking was associated with higher self-reported BMI, higher obesogenic dietary index score, and higher levels of distracted-eating at follow-up. For objective measures, only baseline evening snacking was associated with higher BMI and waist circumference at baseline and follow-up (Table 6). 
Table 4. Associations between baseline snack occasions and self-reported body mass index and related behaviors among PACE adults at baseline $(n=2389)$.

\begin{tabular}{|c|c|c|c|c|c|c|c|c|}
\hline & $\begin{array}{c}\text { BMI } \\
\left(\mathrm{kg} / \mathrm{m}^{2}\right)^{\mathrm{a}} \\
\text { Mean }(95 \% \mathrm{CI})\end{array}$ & $p$-Value & $\begin{array}{c}\text { Fruit \& Veg } \\
\text { (Servings/Day) a } \\
\text { Mean }(95 \% \text { CI) }\end{array}$ & & $\begin{array}{c}\text { Dietary Index } \\
\text { (Times/Week) }^{\text {a }} \\
\text { Mean }(95 \% \text { CI) }\end{array}$ & $p$-Value & $\begin{array}{c}\text { High Distracted-Eating } \\
(\%) \\
\text { Mean }(95 \% \text { CI })\end{array}$ & $p$-Value \\
\hline Morning snack ${ }^{b}$ & & 0.04 & & $<0.0001$ & & $<0.0001$ & & 0.16 \\
\hline 0 & $27.0(26.7,27.4)$ & & $2.5(2.4,2.6)$ & & $2.2(2.1,2.4)$ & & $33.8(30.9,36.8)$ & \\
\hline 1 & $26.7(26.3,27.1)$ & & $2.9(2.8,3.1)$ & & $2.0(1.8,2.2)$ & & $31.1(27.7,34.5)$ & \\
\hline $2+$ & $26.1(25.1,27.2)$ & & $3.3(2.9,3.7)$ & & $1.6(1.2,1.9)$ & & $30.0(20.5,39.5)$ & \\
\hline Mid-day snack ${ }^{c}$ & & 0.68 & & $<0.0001$ & & 0.009 & & 0.02 \\
\hline 0 & $27.0(26.6,27.3)$ & & $2.5(2.4,2.6)$ & & $2.2(2.0,2.4)$ & & $31.1(28.1,34.1)$ & \\
\hline 1 & $26.7(26.3,27.1)$ & & $2.8(2.7,3.0)$ & & $2.0(1.8,2.2)$ & & $33.2(29.9,36.4)$ & \\
\hline $2+$ & $27.2(26.2,28.2)$ & & $3.3(2.9,3.7)$ & & $1.8(1.5,2.2)$ & & $45.3(36.8,53.8)$ & \\
\hline Evening snack ${ }^{d}$ & & $<0.0001$ & & 0.03 & & $<0.0001$ & & $<0.0001$ \\
\hline 0 & $26.5(26.1,26.8)$ & & $2.6(2.5,2.7)$ & & $2.0(1.8,2.2)$ & & $27.9(25.1,30.7)$ & \\
\hline 1 & $27.5(27.0,27.9)$ & & $2.8(2.7,3.0)$ & & $2.3(2.1,2.6)$ & & $39.7(36.3,43.2)$ & \\
\hline $2+$ & $27.9(26.7,29.1)$ & & $2.6(2.2,3.0)$ & & $2.9(2.3,3.5)$ & & $44.4(34.4,54.3)$ & \\
\hline
\end{tabular}

a Log-transformed for analysis; mean values have been back-transformed for presentation. ${ }^{\mathrm{b}}$ Adjusted for age, gender, race/ethnicity, education, manual occupation, leisure-time PA score, and main or light meal reported between 12:00 a.m. and 10:59 a.m. ${ }^{c}$ Adjusted for age, gender, race/ethnicity, education, manual occupation, leisure-time PA score, and main or light meal reported between 11:00 a.m. and 4:29 p.m. ${ }^{\mathrm{d}}$ Adjusted for age, gender, race/ethnicity, education, manual occupation, leisure-time PA score, and main or light meal reported between 4:30 p.m. and 11:59 p.m. 
Table 5. Associations between baseline snack occasions and self-reported body mass index and related behaviors at 2-year follow-up among PACE adults $(n=1151)$.

\begin{tabular}{|c|c|c|c|c|c|c|c|c|}
\hline & $\begin{array}{c}\text { BMI } \\
\left(\mathrm{kg} / \mathrm{m}^{2}\right)^{\mathrm{a}} \\
\text { Mean }(95 \% \mathrm{CI})\end{array}$ & $p$-Value & $\begin{array}{c}\text { Fruit \& Veg } \\
\text { (Servings/Day) a }^{\text {a }} \text { Mean }\left(95 \% \text { CI) }^{\text {a }}\right.\end{array}$ & & $\begin{array}{l}\text { Dietary Index } \\
\text { (Times/Week) }^{\text {a }} \\
\text { Mean }(95 \% \mathrm{CI})\end{array}$ & $p$-Value & $\begin{array}{c}\text { High Distracted-Eating } \\
(\%) \\
\text { Mean }(95 \% \text { CI })\end{array}$ & $p$-Value \\
\hline Morning snack $^{b}$ & & 0.79 & & 0.001 & & 0.54 & & 0.72 \\
\hline 0 & $26.7(26.5,27.5)$ & & $2.8(2.6,3.0)$ & & $1.7(1.5,2.0)$ & & $29.4(25.7,33.0)$ & \\
\hline 1 & $27.1(26.5,27.6)$ & & $3.2(3.0,3.5)$ & & $1.7(1.5,2.0)$ & & $30.5(26.4,34.6)$ & \\
\hline $2+$ & $26.3(24.9,27.9)$ & & $3.5(2.9,4.2)$ & & $1.5(1.1,2.1)$ & & $29.2(16.2,42.1)$ & \\
\hline Mid-day snack $^{c}$ & & 0.95 & & $<0.0001$ & & 0.01 & & 0.22 \\
\hline 0 & $27.0(26.5,27.5)$ & & $2.8(2.6,3.0)$ & & $1.9(1.7,2.1)$ & & $28.4(24.6,32.1)$ & \\
\hline 1 & $26.8(26.3,27.3)$ & & $3.2(3.0,3.5)$ & & $1.5(1.3,1.8)$ & & $31.3(27.2,35.5)$ & \\
\hline $2+$ & $27.9(26.4,29.4)$ & & $3.7(3.1,4.4)$ & & $1.7(1.3,2.3)$ & & $32.8(20.7,44.9)$ & \\
\hline Evening snack ${ }^{\mathrm{d}}$ & & $<0.0001$ & & 0.17 & & $<0.0001$ & & 0.001 \\
\hline 0 & $26.3(25.9,26.8)$ & & $2.9(2.7,3.1)$ & & $1.6(1.4,1.8)$ & & $25.8(22.3,29.3)$ & \\
\hline 1 & $28.0(27.4,28.6)$ & & $3.2(2.9,3.4)$ & & $2.0(1.7,2.2)$ & & $35.7(31.1,40.2)$ & \\
\hline $2+$ & $28.0(26.3,29.9)$ & & $2.9(2.3,3.7)$ & & $2.5(1.8,3.4)$ & & $35.8(21.5,50.1)$ & \\
\hline
\end{tabular}

a Log-transformed for analysis; mean values have been back-transformed for presentation. ${ }^{\mathrm{b}}$ Adjusted for age, gender, race/ethnicity, education, manual occupation, leisure-time PA score, and main or light meal reported between 12:00 a.m. and 10:59 a.m. ${ }^{c}$ Adjusted for age, gender, race/ethnicity, education, manual occupation, leisure-time PA score, and main or light meal reported between 11:00 a.m. and 4:29 p.m. ${ }^{\mathrm{d}}$ Adjusted for age, gender, race/ethnicity, education, manual occupation, leisure-time PA score, and main or light meal reported between 4:30 p.m. and 11:59 p.m. 
Table 6. Associations between baseline snacking occasions and objective measures of body mass index and waist circumference at baseline and 2-year follow-up among PACE adults participating in the intensive assessment subsample.

\begin{tabular}{|c|c|c|c|c|c|c|c|c|}
\hline & & & Baseline & & & & llow-Up & \\
\hline & $\begin{array}{c}\text { BMI }\left(\mathrm{kg} / \mathrm{m}^{2}\right)^{\text {a }} \\
(\mathrm{n}=567) \\
\text { Mean }(95 \% \mathrm{CI})\end{array}$ & $p$-Value & $\begin{array}{c}\text { Waist Circumference }(\mathrm{cm})^{a} \\
(\mathrm{n}=551) \\
\text { Mean }(95 \% \mathrm{CI})\end{array}$ & $p$-Value & $\begin{array}{c}\text { BMI }\left(\mathrm{kg} / \mathrm{m}^{2}\right)^{a} \\
(\mathrm{n}=262) \\
\text { Mean }(95 \% \mathrm{CI})\end{array}$ & $p$-Value & $\begin{array}{c}\text { Waist Circumference }(\mathrm{cm})^{a} \\
(\mathrm{n}=258) \\
\text { Mean }(95 \% \mathrm{CI})\end{array}$ & $p$-Value \\
\hline Morning snack ${ }^{\mathrm{b}}$ & & 0.23 & & 0.21 & & 0.43 & & 0.37 \\
\hline 0 & $28.7(28.0,29.4)$ & & $89.7(87.9,91.5)$ & & $29.1(28.2,30.2)$ & & $87.4(84.9,90.0)$ & \\
\hline 1 & $28.1(27.3,28.8)$ & & $88.4(86.5,90.4)$ & & $28.6(27.5,29.7)$ & & $85.4(82.8,88.2)$ & \\
\hline $2+$ & $28.0(26.0,30.2)$ & & $87.1(82.2,92.4)$ & & $28.3(25.5,31.4)$ & & $86.5(80.0,93.5)$ & \\
\hline Mid-day snack ${ }^{c}$ & & 0.48 & & 0.48 & & 0.11 & & 0.68 \\
\hline 0 & $28.4(27.7,29.1)$ & & $89.6(87.7,91.4)$ & & $28.4(27.4,29.4)$ & & $87.4(84.6,90.4)$ & \\
\hline 1 & $28.4(27.6,29.1)$ & & $88.3(86.5,90.3)$ & & $29.1(28.1,30.2)$ & & $84.9(82.1,87.7)$ & \\
\hline $2+$ & $28.6(26.7,30.7)$ & & $89.3(84.6,94.2)$ & & $31.0(28.1,34.2)$ & & $91.1(84.0,98.7)$ & \\
\hline Evening snack ${ }^{d}$ & & 0.007 & & 0.01 & & 0.001 & & $<0.0001$ \\
\hline 0 & $27.7(27.1,28.4)$ & & $87.7(86.0,89.4)$ & & $28.1(27.2,29.0)$ & & $84.3(82.1,86.6)$ & \\
\hline 1 & $29.2(28.4,30.0)$ & & $90.6(88.6,92.6)$ & & $29.4(28.3,30.6)$ & & $88.6(85.9,91.4)$ & \\
\hline $2+$ & $30.1(27.4,33.0)$ & & $93.386 .7,100.3)$ & & $34.8(31.0,39.1)$ & & $99.5(90.9,108.9)$ & \\
\hline
\end{tabular}

${ }^{a}$ Log-transformed for analysis; mean values have been back-transformed for presentation. ${ }^{\mathrm{b}}$ Adjusted for age, gender, race/ethnicity, education, manual occupation, leisure-time PA score, and main or light meal reported between 12:00 a.m. and 10:59 am. ${ }^{c}$ Adjusted for age, gender, race/ethnicity, education, manual occupation, leisure-time PA score, and main or light meal reported between 11:00 a.m. and 4:29 p.m. ${ }^{\mathrm{d}}$ Adjusted for age, gender, race/ethnicity, education, manual occupation, leisure-time PA score, and main or light meal reported between 4:30 p.m. and 11:59 p.m. 


\section{Discussion}

We present associations between snacking patterns, several obesogenic behaviors, and obesity. The snacking patterns may be considered analogous to extra eating because the analysis adjusted for consuming a meal during the same time-period. Notably, we have found that associations depend on the timing of the snack occasion. Specifically, evening snacking was found to be an obesogenic behavior. Higher levels of evening snacking were associated with higher BMI over repeated self-reported and objective measures at baseline and follow-up. Given the high correlation between self-reported and measured BMI at baseline, consistency in these findings is significant. Furthermore, this relationship was also consistent for repeated measures of waist circumference as well as two measures of obesity-related behaviors, namely the obesogenic dietary index [18] and distracted-eating [22,23]. Interestingly, these patterns of associations were not evidenced for morning or mid-day snack occasions. Higher levels of morning or mid-day snacking were not associated with obesity, yet were associated with higher fruit and vegetable intake. Higher mid-day snacking was also associated with lower obesogenic dietary index scores. To our knowledge, these are the first findings to present associations between timing of snack occasion and measures of obesity as well as related behaviors while adjusting for other meals consumed in the same period.

Our findings are consistent with some $[4-6,26]$, but not all $[27,28]$, studies noting associations between increased snack frequency and obesity among adults. Our findings are also consistent with others noting positive relationships between evening snacking and obesity $[6,9,26]$ as well as negative relationships between morning and mid-day snacking and obesity [29]. These findings are supported by studies that have noted higher energy-density of snacks overall among U.S. adults [30] as well as higher energy-density of evening snacks and lower energy-density of morning snacks among French adults [31]. Mechanistically, there is a growing body of evidence noting the importance of circadian timing of food intake to maintain proper metabolic function and body weight regulation [32,33]. We extend previous results by not only adjusting for multiple individual characteristics including demographics and physical activity, but by accounting for other eating occasions during the same period as well.

Our findings are also consistent with observational studies noting positive associations between distracted-eating and subsequent snack intake [34] as well as experimental studies that either introduce eating mindfulness [35,36] or eating distractions [36]. Few studies have examined snacking in relation to fruit and vegetable intake. Kant and colleagues recently reported that fruit, not vegetable, consumption was higher on days when people snacked compared to days when people did not snack [37]. To our knowledge, there are no other studies that have examined relationships between snacking and fast food or soda intake. Comparisons to studies reporting associations between snacking and diet quality may be most relevant, although this body of evidence is also relatively small and also mixed [38-40]. It is possible that mixed findings are due to differences in the composition of snack foods which likely vary across populations defined by age $[39,40]$ as well as culture.

There are several strengths to this study including: a rigorous statistical approach including multivariable adjustment, use of repeated measures, use of physical measurements for weight, height, and waist circumference, and use of a meal-pattern grid for defining eating occasions. This method [13,14] follows best practices for defining eating occasions [28], specifically, allowing the respondent to distinguish between meals and snacks as well as report the time of eating occasions. This study also includes some limitations. We must acknowledge that we cannot disentangle snack timing from snack composition. It is possible, for example, that it is not really evening snacking that is associated with obesity, but the composition of the snacks that are consumed at that time. We also did not collect information about sleep or other measures of circadian disruption which could have validated the importance of snack timing in these findings. BMI and waist circumference may also not be the most accurate measures of obesity, with different errors as well as implications for different race/ethnic groups. We are also limited by attrition of participants over follow-up as well as a smaller sample size for participants providing physical measurements, each of which not only lowers analytic 
power but also threatens generalizability to the initial total group of baseline responders. Finally, our respondent group itself may not be generalizable to the U.S. general population given its composition of mostly White working adults.

\section{Conclusions}

Snacking is an important behavior to modify for obesity prevention, although additional dimensions of this behavior need to be considered to produce effective and consistent messaging [41]. Clearly, we note that paying attention to the frequency and timing of snacking is important for obesity prevention. However, we must acknowledge that snack composition is also important given associations with specific dietary behaviors for morning, mid-day, and evening snacks. Reducing evening snacks, and therefore intake of more energy-dense foods, may be another important and simple message for population-level obesity prevention efforts.

Supplementary Materials: The following are available online at http://www.mdpi.com/2072-6643/11/10/2320/s1, Figure S1: Adaptation of eating pattern assessment used in the Promoting Activity and Changes in Eating (PACE) study, 2005-2009.

Author Contributions: W.E.B. and S.A.A.B. contributed equally to the conceptualization and methodology of this study. W.E.B. took the lead on formal analyses, original manuscript draft preparation and revision. S.A.A.B. was responsible for funding acquisition, project administration, and review and editing of the manuscript.

Funding: The Promoting Activity and Changes in Eating (PACE) Study was funded by National Heart, Lung, and Blood Institute, grant number R01 HL079491. Dr. Barrington was supported by the Biobehavioral Cancer Prevention and Control Training (BCPT) Program funded by the National Cancer Institute, grant number T32 CA092408.

Conflicts of Interest: The authors declare no conflict of interest.

\section{References}

1. Flegal, K.M.; Kruszon-Moran, D.; Carroll, M.D.; Fryar, C.D.; Ogden, C.L. Trends in Obesity among Adults in the United States, 2005 to 2014. JAMA 2016, 315, 2284-2291. [CrossRef] [PubMed]

2. Lemmens, V.E.; Oenema, A.; Klepp, K.I.; Henriksen, H.B.; Brug, J. A systematic review of the evidence regarding efficacy of obesity prevention interventions among adults. Obes. Rev. 2008, 9, 446-455. [CrossRef] [PubMed]

3. Leech, R.M.; Worsley, A.; Timperio, A.; McNaughton, S.A. Understanding meal patterns: Definitions, methodology and impact on nutrient intake and diet quality. Nutr. Res. Rev. 2015, 28, 1-21. [CrossRef] [PubMed]

4. Bellisle, F. Meals and snacking, diet quality and energy balance. Physiol. Behav. 2014, 134, 38-43. [CrossRef] [PubMed]

5. Kahleova, H.; Lloren, J.I.; Mashchak, A.; Hill, M.; Fraser, G.E. Meal Frequency and Timing Are Associated with Changes in Body Mass Index in Adventist Health Study 2. J. Nutr. 2017, 147, 1722-1728. [CrossRef] [PubMed]

6. Berg, C.; Forslund, H.B. The Influence of Portion Size and Timing of Meals on Weight Balance and Obesity. Curr. Obes. Rep. 2015, 4, 11-18. [CrossRef] [PubMed]

7. Hess, J.M.; Jonnalagadda, S.S.; Slavin, J.L. What Is a Snack, Why Do We Snack, and How Can We Choose Better Snacks? A Review of the Definitions of Snacking, Motivations to Snack, Contributions to Dietary Intake, and Recommendations for Improvement. Adv. Nutr. 2016, 7, 466-475.

8. Mesas, A.E.; Munoz-Pareja, M.; Lopez-Garcia, E.; Rodriguez-Artalejo, F. Selected eating behaviours and excess body weight: A systematic review. Obes. Rev. 2012, 13, 106-135. [CrossRef]

9. McCrory, M.A.; Shaw, A.C.; Lee, J.A. Energy and Nutrient Timing for Weight Control: Does Timing of Ingestion Matter? Endocrinol. Metab. Clin. N. Am. 2016, 45, 689-718. [CrossRef]

10. Liebman, M.; Pelican, S.; Holmes, B.; Wardlaw, M.K.; Melcher, L.M.; Liddil, A.C.; Paul, L.C.; Dunnagan, T.; Haynes, G.W. Dietary intake, eating behavior, and physical activity-related determinants of high body mass index in rural communities in Wyoming, Montana, and Idaho. Int. J. Obes. Relat. Metab. Disord. 2003, 27, 684-692. [CrossRef] 
11. Lowe, M.R.; Doshi, S.D.; Katterman, S.N.; Feig, E.H. Dieting and restrained eating as prospective predictors of weight gain. Front. Psychol. 2013, 4, 577. [CrossRef] [PubMed]

12. Scherwitz, L.; Kesten, D. Seven eating styles linked to overeating, overweight, and obesity. Explore 2005, 1, 342-359. [CrossRef] [PubMed]

13. Berteus Forslund, H.; Lindroos, A.K.; Sjostrom, L.; Lissner, L. Meal patterns and obesity in Swedish women-a simple instrument describing usual meal types, frequency and temporal distribution. Eur. J. Clin. Nutr. 2002, 56, 740-747. [CrossRef] [PubMed]

14. Berteus Forslund, H.; Torgerson, J.S.; Sjostrom, L.; Lindroos, A.K. Snacking frequency in relation to energy intake and food choices in obese men and women compared to a reference population. Int. J. Obes. 2005, 29, 711-719. [CrossRef] [PubMed]

15. Beresford, S.A.; Locke, E.; Bishop, S.; West, B.; McGregor, B.A.; Bruemmer, B.; Duncan, G.E.; Thompson, B. Worksite study promoting activity and changes in eating (PACE): Design and baseline results. Obesity 2007, 15 (Suppl. 1), 4S-15S. [CrossRef]

16. Thompson, F.E.; Midthune, D.; Subar, A.F.; McNeel, T.; Berrigan, D.; Kipnis, V. Dietary intake estimates in the National Health Interview Survey, 2000: Methodology, results, and interpretation. J. Am. Diet. Assoc. 2005, 105, 352-363. [CrossRef]

17. Thompson, F.; Byers, T. Dietary Assessment Resource Manual. J. Nutr. 1994, 124, 2245S-2317S.

18. Barrington, W.E.; Beresford, S.A.A. Applying Multiple Statistical Methods to Derive an Index of Dietary Behaviors Most Related to Obesity. Am. J. Epidemiol. 2018, 187, 1559-1569. [CrossRef]

19. Dave, J.M.; An, L.C.; Jeffery, R. Relationship of attitudes toward fast-food and frequency of fast-food intake in adults. Obesity 2009, 17, 1164-1170. [CrossRef]

20. Rosenheck, R. Fast food consumption and increased caloric intake: A systematic review of a trajectory towards weight gain and obesity risk. Obes. Rev. 2008, 9, 535-547. [CrossRef]

21. French, S.A.; Harnack, L.; Jeffery, R.W. Fast food restaurant use among women in the Pound of Prevention study: Dietary, behavioral and demographic correlates. Int. J. Obes. Relat. Metab. Disord. 2000, 24, 1353-1359. [CrossRef] [PubMed]

22. Framson, C.; Kristal, A.; Schenk, J.M.; Littman, A.; Zeliadt, S.; Benitez, D. Development and validation of the Mindful Eating Questionnaire. J. Am. Diet. Assoc. 2009, 109, 1439-1444. [CrossRef] [PubMed]

23. Wansink, B. Environmental factors that increase the food intake and consumption volume of unknowing consumers. Annu. Rev. Nutr. 2004, 24, 455-479. [CrossRef] [PubMed]

24. Godin, G.; Shephard, R. A simple method to assess exercise behavior in the community. Can. J. Appl. Sport Sci. 1985, 10, 141-146. [PubMed]

25. Amireault, S.; Godin, G. The Godin-Shephard leisure-time physical activity questionnaire: Validity evidence supporting its use for classifying healthy adults into active and insufficiently active categories. Percept. Mot. Ski. 2015, 120, 604-622. [CrossRef] [PubMed]

26. Okada, C.; Imano, H.; Muraki, I.; Yamada, K.; Iso, H. The Association of Having a Late Dinner or Bedtime Snack and Skipping Breakfast with Overweight in Japanese Women. J. Obes. 2019, 2019, 2439571. [CrossRef]

27. Vatanparast, H.; Islam, N.; Patil, R.P.; Shafiee, M.; Smith, J.; Whiting, S. Snack Consumption Patterns among Canadians. Nutrients 2019, 11, 1152. [CrossRef] [PubMed]

28. St-Onge, M.P.; Ard, J.; Baskin, M.L.; Chiuve, S.E.; Johnson, H.M.; Kris-Etherton, P.; American Heart Association Obesity Committee of the Council on Lifestyle and Cardiometabolic Health; Council on Cardiovascular Disease in the Young; Council on Clinical Cardiology; Stroke Council; et al. Meal Timing and Frequency: Implications for Cardiovascular Disease Prevention: A Scientific Statement From the American Heart Association. Circulation 2017, 135, e96-e121. [CrossRef]

29. Aparicio, A.; Rodriguez-Rodriguez, E.E.; Aranceta-Bartrina, J.; Gil, Á.; González-Gross, M.; Serra-Majem, L.; Varela-Moreiras, G.; Ortega, R.M. Differences in meal patterns and timing with regard to central obesity in the ANIBES ('Anthropometric data, macronutrients and micronutrients intake, practice of physical activity, socioeconomic data and lifestyles in Spain') Study. Public Health Nutr. 2017, 20, 2364-2373. [CrossRef]

30. Krok-Schoen, J.L.; Jonnalagadda, S.S.; Luo, M.; Kelly, O.J.; Taylor, C.A. Nutrient Intakes from Meals and Snacks Differ with Age in Middle-Aged and Older Americans. Nutrients 2019, 11, 1301. [CrossRef]

31. Si Hassen, W.; Castetbon, K.; Tichit, C.; Péneau, S.; Nechba, A.; Ducrot, P.; Lampuré, A.; Bellisle, F.; Hercberg, S.; Méjean, C. Energy, nutrient and food content of snacks in French adults. Nutr. J. 2018, 17, 33. [CrossRef] [PubMed] 
32. Gallant, A.; Lundgren, J.; Drapeau, V. Nutritional Aspects of Late Eating and Night Eating. Curr. Obes. Rep. 2014, 3, 101-107. [CrossRef] [PubMed]

33. Kessler, K.; Pivovarova-Ramich, O. Meal Timing, Aging, and Metabolic Health. Int. J. Mol. Sci. 2019, $20,1911$. [CrossRef]

34. Robinson, E.; Aveyard, P.; Daley, A.; Jolly, K.; Lewis, A.; Lycett, D.; Higgs, S. Eating attentively: A systematic review and meta-analysis of the effect of food intake memory and awareness on eating. Am. J. Clin. Nutr. 2013, 97, 728-742. [CrossRef] [PubMed]

35. Robinson, E.; Kersbergen, I.; Higgs, S. Eating 'attentively' reduces later energy consumption in overweight and obese females. Br. J. Nutr. 2014, 112, 657-661. [CrossRef]

36. Higgs, S. Manipulations of attention during eating and their effects on later snack intake. Appetite 2015, 92, 287-294. [CrossRef] [PubMed]

37. Kant, A.K.; Graubard, B.I. Within-person compensation for snack energy by US adults, NHANES 2007-2014. Am. J. Clin. Nutr. 2019, 109, 1145-1153. [CrossRef]

38. Leech, R.M.; Livingstone, K.M.; Worsley, A.; Timperio, A.; McNaughton, S.A. Meal Frequency but Not Snack Frequency Is Associated with Micronutrient Intakes and Overall Diet Quality in Australian Men and Women. J. Nutr. 2016, 146, 2027-2034. [CrossRef]

39. Murakami, K.; Livingstone, M.B. Associations between Meal and Snack Frequency and Diet Quality in US Adults: National Health and Nutrition Examination Survey 2003-2012. J. Acad. Nutr. Diet. 2016, 116, 1101-1113. [CrossRef]

40. Si Hassen, W.; Castetbon, K.; Peneau, S.; Tichit, C.; Nechba, A.; Lampuré, A.; Bellisle, F.; Hercberg, S.; Méjean, C. Socio-economic and demographic factors associated with snacking behavior in a large sample of French adults. Int. J. Behav. Nutr. Phys. Act. 2018, 15, 25. [CrossRef]

41. Potter, M.; Vlassopoulos, A.; Lehmann, U. Snacking Recommendations Worldwide: A Scoping Review. Adv. Nutr. 2018, 9, 86-98. [CrossRef] [PubMed]

(C) 2019 by the authors. Licensee MDPI, Basel, Switzerland. This article is an open access article distributed under the terms and conditions of the Creative Commons Attribution (CC BY) license (http://creativecommons.org/licenses/by/4.0/). 